
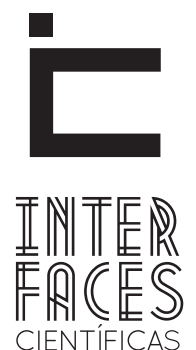

EDUCAÇÃO

ISSN IMPRESSO 2316-333X

ISSN ELETRÔNICO 2316-3828

\title{
FORMAS ACTUALES DEL CONTROL SOCIAL: IMPACTOS EN LA EDUCACIÓN SOCIAL. REFLEXIONES DESDE LA PEDAGOGÍA SOCIAL
}

Violeta Maria Nuñez Perez ${ }^{1}$

\section{RESUMEN}

La exposición articula dos grandes cuestiones que se juegan en este momento histórico en el estado español: 1) Las políticas sociales de control que responden al resurgimiento del discurso higienista. Se mostrarán algunas de sus derivas neo higienistas en el campo de las ciencias y las políticas sociales. En el campo de la pedagogía social se dará cuenta del entrenamiento de los operadores del control social, quienes vienen a sustituir a los profesionales de la educación social; 2) Posibilidades y márgenes desde una Pedagogía Social crítica: aportaciones para ejercicios de resistencia y desbordamiento de cauces previstos, tanto en la institución universitaria como en el campo social. Concluiremos que es posible perforar ese talud neoliberal que se pretende monolítico y abrir, allí donde parece altamente improbable, otros espacios de invención e intercambio cultural y educativo.

\section{PALABRAS CLAVE}

Políticas neohigienistas. Tecnopoder. Operadores. Pedagogía Social. 


\section{RESUMO}

A exposição articula duas grandes questões em jogo neste momento histórico no Estado espanhol: 1) As políticas de controle social que respondem ao ressurgimento do discurso higienista. Serão mostradas algumas de suas derivações neohigienistas no campo da ciência e da política social. No campo da pedagogia social vai-se perceber o treinamento de operadores de controle social, que vêm para substituir o profissional da educação social; 2) Oportunidades e margens desde a Pedagogia Social crítica: subsídios para exercícios de resistência e transbordamento dos canais previstos, tanto na universidade como no campo social. Concluiremos que é possível perfurar esse penhasco neoliberal que se pretende monolítico e abrir, ali onde parece altamente improvável outros espaços de invenção e de intercâmbio cultural e educativo.

\section{PALAVRAS-CHAVE}

Políticas neo-higienistas. Tecnopoder. Operadores. Pedagogia Social.

\section{ABSTRACT}

The text articulates two major issues at play in this historic moment in the Spanish state: 1) Social control policies respond to the resurgence of the hygienist's discourse. Some of their proposals in the field of social science and social policy will be shown. In the field of social pedagogy will realize the training of social control operators that come to replace professional social education; 2) Opportunities and margins from a critical Social Pedagogy: contributions to resistance exercises and overflow channels

provided both in the university and in the social field. Conclude that it is possible to drill the neoliberal discourse that aims monolithic, there where it seems highly unlikely, other areas of invention and cultural and educational exchange.

\section{KEYWORDS}

Neo-Hygienists Policies. Techno-Power. Operators. Social Pedagogy. 


\section{PRESENTACIÓN DEL TEXTO}

Quiero señalar que este texto ${ }^{2}$ responde al lugar desde el cual lo enuncio: profesora de la universidad de Barcelona; no pretende pues, esta exposición, constituirse en "LA" verdad, sino contribuir a la búsqueda de verdades que, en diferentes espacios geográficos, políticos y teóricos vamos emprendiendo, pese al imperativo neoliberal que busca erradicar toda reflexión crítica sobre la realidad ${ }^{3}$.

Voy a centrar la exposición en dos grandes cuestiones que entiendo son las centrales, en este momento histórico y en el estado español, en lo que atañe a las políticas sociales de control y a las funciones de la educación y acción social que de ellas se desprenden:

1) A modo de cuerpo central del artículo: resurgimiento del higienismo y sus derivas neohigienistas en el campo de las ciencias y las políticas sociales, haciendo hincapié en su empuje para la formación de operadores (ya no profesionales) del campo social;

2) A modo de conclusión y nuevas aperturas desde una Pedagogía Social crítica: aportaciones para ejercicios de resistencia y desbordamiento de cauces previstos.

\section{RESURGIMIENTO DEL HIGIENISMO Y SUS DERIVAS}

El pasaje del capitalismo industrial al informacional, registrado a partir del último tercio del XX,

2. Retoma el trabajo presentado en las Jornadas de la Universidad de Castilla- La Mancha, en abril de 2011 y que sigo trabajando en el grupo de investigación interuniversitario Tentativas.

3. En cuanto a aportaciones críticas sobre el campo de la Pedagogía / Educación social, consultar: MOYANO, S.; FRYD, P. (2012). "El escenario pedagógico de la educación social”. En Pedagógica n² 2, abril 2012. Departamento de Pedagogía, Política y Sociedad de la Universidad dela República Oriental del Uruguay. Disponible en: <http://pedagogica.fhuce.edu.uy/images/numero2/ rp 2 d moyano fryd.pdf>. GARCÍA MOLINA, J. (2013). Indisciplinar la Pedagogía Social. Virtualidades y obstáculos. Educatio Siglo XXI, v.31, n.2, p.35-55. Disponible en: <http://revistas.um.es/educatio/issue/view/11741/showToc>. comporta cambios significativos tanto en el concepto mismo de ciudadanía como en su carácter de tejido conectivo de lo social. En esta nueva modalidad del capitalismo, el ejercicio del poder tiende a in-visibilizarse ante la mirada del gran público: una red tupida pero descentralizada, flexible, capaz de evadir los controles territoriales de lo político y de imponer sus normas predadoras, todo esto llamado eufemísticamente, presiones de los mercados. Una suerte de licuefacción o, tal como lo plantea Zygmunt Bauman: modernidad líquida.

La volatilización del poder económico produce efectos de fragmentación en los territorios, al sustraer del ámbito de sus leyes el actual núcleo duro (informacional) del capitalismo de rapiña, tal como lo denomina Helmut Schmidt.

Quitado el que fuera el marco de la organización del capitalismo industrial en el pasado siglo, es decir, el pacto social del capital y la fuerza de trabajo (BAUMAN, 2000), los sectores sociales quedan disgregados, atomizados. Esta fragmentación supone el quiebre de las antiguas solidaridades, el resquebrajamiento de la ciudadanía, dejando a los sujetos librados a su suerte. Pero ello no significa descontrol, sino nuevas modalidades de control: ¿qué modalidades de control pueden ejercerse en la modernidad líquida?

La solución es de una simpleza excepcional, a saber: que cada cual vigile por sí mismo. Lo social se des-articula y cada cual queda expuesto a las consecuencias de esa fragmentación. Aquí se ubica el por qué del éxito de los libros de autoayuda, la promoción del sentimiento por encima de otra consideración, el imperativo a serfeliz sin límite, de "tener" autoestima etc.

Ahora bien, es necesario también el control de las poblaciones que se definan como sectores potencialmente peligrosos, es decir, que no comulguen con el modelo de felicidad que el utilitarismo vende como estilo de vida. 
En este punto es donde el recurso al neohigienismo hace su entrada. No son nuevas las relaciones entre la tecnología y el ejercicio del poder de control, hay que referirse como antecedente a los estudios de Foucault (1981), acerca de lo que llamó sociedad disciplinar.

Hoy, el tecnopoder remite a la noción de sociedad del control. Es una definición que, a partir de los trabajos de Foucault, retrabajan diferentes autores. Voy a centrarme en la aportación que A. Negri ${ }^{4}$ realiza en 2002, en el libro Imperio. Las TIC devienen el mecanismo fundamental (y acaso único), de la estandarización que el discurso del poder hoy necesita: estandarización de formas, de representaciones, de lenguajes, de inerfaces.

En los campos de las políticas sociales, dicha estandarización borra las subjetividades, diluyendo a los sujetos en flujos estadísticos de elementos idénticos. Esto supone un fuerte empuje para que las disciplinas sociales tiendan a configurarse como espacio facilitador de instrumentos eficaces para ese ejercicio de poder.

En efecto, el discurso hegemónico en las ciencias sociales, promueve la colaboración de instancias académicas y profesionales con empresas públicas, privadas $y / 0$ del tercer sector, para elaborar y poner en práctica nuevos y más eficaces protocolos para gestionar los llamados perfiles poblacionales, en nombre de la prevención. Se estructuran así verdaderos espacios concentracionarios (para emplear el concepto de G. Agamben).

Fue Robert Castel quien planteó el tema de la gestión social de las poblaciones ${ }^{5}$ desde la crítica a la idea de "prevención". Esta, como bien se sabe, es particu-

4. Hay un libro más reciente, también escrito en colaboración con Michael Hardt que retoma algunas de estas consideraciones. HARDT, M. / NEGRI, A. (2011): Commonwelth: el proyecto de una revolución del común. Madrid: Akal.

5. En efecto, en su libro La gestión de los riesgos, publicado en Les Editions de Minuit, Paris, 1981 y editado en castellano en 1984, por Anagrama, Castel advierte la emergencia de la tecnología en el campo del control de poblaciones: actividades periciales, de evaluación y distribución, como estrategias inéditas de tratamiento de los problemas sociales. larmente aplicada en aquellos sectores sociales que se seleccionan para los planes y proyectos de la llamada intervención social. La prevención convoca a intervenir en la vida de otro: allí donde se considera que puede representar un peligro para sí mismo y/o para la sociedad, extremo que muestra la película de ciencia ficción Minority Report, dirigida por Steven Spielberg (2002) y basada en un relato corto de 1956 de Philip K. Dick.

Las políticas preventivas no son ingenuas, aunque sí suelen serlo sus operadores, ésos que antes eran profesionales, pues su formación podía habilitarlos para el análisis crítico de la realidad: una cierta distancia entre la demanda del mercado y el discurso pedagógico (en términos de teoría y praxis).

La política preventiva establece categorías diferenciales de individuos, de allí que la denomine: furia entomológica 6 .

Cualquier diferencia puede dar lugar a un perfil poblacional: familias desestructuradas, madres solteras, ex-delincuentes, desocupados, adolescentes embarazadas, jóvenes peligrosos, inmigrantes.

Luego viene la gestión de los mismos, a través procesos de distribución y circulación en circuitos especiales: recorridos sociales bien definidos para esos perfiles poblacionales ${ }^{7}$.

Señala Touraine (2005) que vivimos el final de la representación "social" de las personas, es decir, de su consideración según el lugar que ocupan en la sociedad:

la representación de un personaje consiste en indicar la función "social” y el entorno "social" de ese personaje, y sus características personales se perciben tanto mejor cuando los marcos sociales de quien es representado están indicados de forma más clara. (TOURAINE, 2005, p. 19).

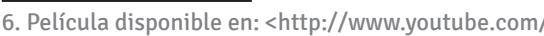
watch?v=0VRtTveygK0>.

7. Una buena ilustración del tema la ofrece la película de Andrew Niccol (1997): GATTACA. 
Sin embargo, podemos ubicar la excepción: las personas sujetas a control directo a través de los dispositivos de gestión poblacional, donde pierden su singularidad en aras de su representación social ${ }^{8}$.

Touraine también plantea (2005, p. 19) que "Las ciencias sociales tienen un retraso particularmente grave que colmar. Demasiado a menudo hablan todavía de la realidad social en términos que ya no corresponden al modelo cultural en que vivimos []".

Vamos a detenernos brevemente y preguntarnos

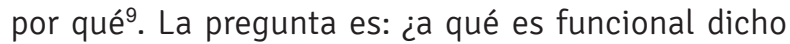
retraso? Podemos constatar que, en Europa y Estados Unidos, los estudios e investigaciones en el campo social, realizados desde los parámetros evaluativos al uso de las universidades, cuentan, en su gran mayoría, con los siguientes atributos:

- sostienen, como bien lo señala Touraine, modelos anacrónicos de representación social;

- son de carácter empírico y pensamiento circular: parten de una hipótesis que se convalida estadísticamente (Teorema de Thomas) ${ }^{10}$;

- ratifican la gestión de lo dado proponiendo la optimización de los medios, en términos de una gestión más ágil, más eficiente.

Estos estudios e investigaciones son promovidos, evaluados y subvencionados por instancias evaluadoras que tramitan el aval científico conforme a la nueva lógica del capitalismo. Dichas investigaciones

8. Película disponible en: <http://www.youtube.com/watch?v=P_X500l2rhQ>.

9. Desde Kant sabemos que interrogarnos acerca del por qué es realizar un ejercicio crítico: interrogarnos acerca de lo que está detrás o fundamenta aquello que aparece.

10. William Thomas en su libro de 1928: The child in America: Behavior problems and programs. New York: Knopf (p.571-572), formula lo que desde entonces se conoce como: Teorema de Thomas: "[...] if men define situations as real, they are real in their consequences" [Si las personas definen las situaciones como reales, éstas son reales en sus consecuencias] ya no apuntan al cuestionamiento de lo que aparece. De allí que lo relevante sea el puro estudio empírico.

En políticas sociales, esto funciona como justificación de las medidas y los dispositivos de control. Es un cúmulo de certezas que habilita a los servicios, a los programas y a los operadores para la gestión diferencial de personas, agrupadas en circuitos precarios según la representación social que las define: familias desestructuradas, jóvenes peligrosos, menores en riesgo, aunque se pretenda que esas representaciones son categorías científicas llamadas "perfiles".

La formación universitaria de los educadores y trabajadores sociales no es ajena a estos avatares, bien al contrario: es un campo de ejercicio de las mismas premisas. Las mismas que el titulado encontrará, a su vez, en la gran mayoría de las ofertas de trabajo. Lo inquietante es la circularidad de estos recorridos, donde la reflexión crítica queda excluida y se abren cursos de dotación de sentidos peregrinos de representación social de los sujetos-objeto de las intervenciones.

Ese retraso del que habla Touraine es la marca de diversas formaciones universitarias ligadas al campo de las políticas sociales. En los currículos universitarios, ese retraso podemos relacionarlo con el vaciamiento de saberes en sentido fuerte, que se conjuga con una fuerte precarización del profesorado.

Esto reenvía a la imposibilidad de hacer escuela, de sostener espacios de confrontación y discusión epistemológica, para instaurar el "pastiche" que denuncia Touraine.

¿Qué transmisión puede tener lugar en estas condiciones? ¿Qué formación construye al estudiante, abocado a discutir informaciones u opiniones, sin confrontación con saberes disciplinares estructurados?

El discurso hegemónico prima la inmediatez de uso de productos. Ello lleva, en nuestro campo, a una competición en pos de imponer (en el mercado 
de las organizaciones que contratan a educadores y trabajadores sociales), protocolos que mejor, más rápido y más barato, garanticen su función. $Y$, a las carreras universitarias (hablo de la generalidad, no de sus honrosas excepciones), a entrenar a sus estudiantes en el manejo y evaluación de tales protocolos, a fin de "garantizar salidas profesionales". Ya no se trata de interrogarse acerca de dicha función, sino de realizarla con eficiencia. Por ello se prima el entrenamiento en competencias, no la construcción de saberes.

No importa a qué estamos contribuyendo, ni siquiera qué estamos construyendo, sino que el imperativo es hacer. Y hacer rápido y bien. Aunque desde Aristóteles sabemos que en educación no se trata de un hacer que aboca a resultados ya previstos, sino de una acción abierta a la incertidumbre o "praxis".

Claro que ¿para qué la "formación universitaria", hoy, se detendría en Aristóteles, o en Hannah Arendt o en Foucault o Agamben, que retomaron y actualizaron esos viejos conceptos? ¿Para qué enredarse en discusiones de fondo, cuando el imperativo es: hacer, hacer, hacer, en la pulida superficie de la pantalla de un presente continuo?

La pregunta que emerge aquí como necesaria es ¿por qué en los estudios universitarios, en el campo de las ciencias sociales, se aplican criterios que no se corresponden ni con su objeto de estudio ni con las prácticas profesionales específicas en las que deberían formar?

La liquidación de esta especificidad se recubre con los nuevos nombres del vaciamiento cultural:

- Las llamadas competencias de nombres abstrusos (cognitivas, metodológicas, de capacidad emprendedora, etc.) que operan la infantilización de los estudiantes universitarios bajo una supuesta cientificidad de una supuesta pedagogía;
- La búsqueda de verbos "observables” para formular objetivos (se trata de listas categorizadas según el imperativo infantilizador también para el profesorado);

- Las asignaturas (iiy los máster!!) orientados a la intervención, a la detección de riesgos y a la evaluación de poblaciones en dichos estándares, a la publicación de esos datos empíricos en revistas anglosajonas.

Los estudiantes, en el proceso de vaciamiento cultural, han devenido alumnos. La parvulización $n^{11}$ de la universidad es un hecho que atañe (como ya se ha indicado), a la infantilización del estudiantado, a la consideración de los jóvenes como desprovistos de toda cultura académica, y reacios a la misma. iiFinalmente lo lograremos!! Pero los profesores también se "parvulizan" a partir del vaciamiento de recursos teóricos y práxicos.

Claro que no es posible el trabajo de transmisión / apropiación hoy en los mismos términos que unos lustros atrás. Claro que hay que innovar. El problema es desde qué premisas y en qué dirección se promueve.

El discurso hegemónico en las universidades se pretende sin fisuras. Desde esa representación busca el consenso. Así, los profesores contribuyen a la difusión e implementación del discurso académico-empresarial del tecnopoder, de su lógica en el campo social y de su expresión política, el neohigienismo.

Para sintetizar, lo que hemos ido reseñando dibuja un paisaje más o menos homogéneo de la formación de los profesionales sociales en el estado español que los transforma en operadores neohigienistas del tecnopoder. Es decir, operadores de la lógica del capitalismo informacional en las tareas del control social.

11. Párvulo: dejamos de lado la acepción de pequeño, niño. Recogemos la de "que sabe poco o es fácil de engañar". También "parvulez": "pequeñez, simplicidad. Cosa de poco momento, de escasa importancia". Enciclopedia Espasa Calpe (1988), Madrid. Tomo XLII. 
Entendemos que es imperiosa la renovación de las ciencias sociales y su actualización a la modernidad líquida, pero disentimos radicalmente de la ideología y de la perspectiva tecnocientífica desde las que se está realizando. Sin embargo, este núcleo fuerte se resguarda de toda discusión.

Hoy el neohigienismo ${ }^{12}$ es claramente empresarial. De donde costes y beneficios; evaluación de calidad del servicio por parte de los clientes; eficiencia y control de procesos en términos de tiempos y resultados numéricos; por citar algunos ejemplos, son los significantes que puntúan el campo social.

Ciertamente, el trabajo del educador/trabajador social queda cada vez más supeditado a la aplicación de protocolos, que estandarizan tanto las tareas de gestión poblacional como su evaluación, re-alimentando el procesamiento informático de los datos y relanzando el circuito, amparado en la estadística que él mismo genera.

Otro asunto central es la redefinición del recurso moralizador para los sectores considerados por el higienismo decimonónico como "degenerados". La primera pregunta es, por tanto, cómo se llaman hoy los "degenerados" de ayer. Lo sabemos. Reciben el nombre de prescindibles. Ya no es necesario mantener disponibles a todos los sectores desocupados, pues no serán reabsorbidos. "Lo que se lleva” en esta nueva sociedad del control es la propuesta de innumerables actividades que los propios operadores del control llevan a cabo: el ocio; la subvención con contraprestaciones; las ocupaciones precarias; los estudios devaluados a contención; el entretenimiento ${ }^{13}$ de niños, adolescentes, jóvenes, adultos y $3^{a}$ edad en actividades sin articulación con la producción y el disfrute de la cultura.

12. A diferencia del decimonónico, que era el de la moral burguesa. 13. En relación a la ilusión y escenificación a la que apunta Guy Debord en La sociedad del espectáculo o a la cultura como simulacro, en palabras de Baudrillard.

\section{DESDE LA PEDAGOGÍA SOCIAL: APORTACIONES PARA EJERCICIOS DE RESISTENCIA Y DESBORDAMIENTO DE CAUCES PREVISTOS}

La educación social, considerada desde el paradigma crítico estructural (en el que me inscribo), es una práctica que pone en acto una justicia redistributiva: redistribuir herencias culturales a sujetos particulares, legitimando sus intereses, propiciando sus producciones y mostrando vías posibles al intercambio cultural, la promoción, la creación.

"Sobre las ruinas de la sociedad sacudida y destruida por la globalización surge un conflicto central que opone, por un lado, fuerzas no sociales reforzadas por la propia globalización y, por otro, al sujeto privado de los sustentos sociales, que han sido liquidados" (TOURAINE, 2005). Y es el sujeto quien se esfuerza por crear instituciones y reglas de derecho que podrían dar sustento a su libertad y creatividad.

Nos encontramos con un cambio profundo, no sólo de enfoque o de marco de referencia, sino un cambio de cultura, ya que el paso a una visión cultural de nuestras vidas implica de por sí una transformación en la cultura misma y, por tanto, de los modos de pensar y actuar también en educación y acción social.

La preocupación preponderante en nuestra época es la capacidad de crearse, de legitimarse, es decir, construirse como "sujeto".

El retorno, un tanto inesperado, del concepto de derechos humanos puede, para Touraine (2005), ayudar la cuestión del sujeto en este momento histórico. Los derechos humanos aparecen cuando tratamos de analizar qué es lo que mueve a la gente, qué es lo que consideramos más importante, y que resulta ser algo que se expresa "en términos de exigencia de respeto, de lucha contra la humillación o de deseo de ser tratado como un ser humano, aplicando derechos individuales de tipo universal" (TOURAINE, 2005, p.58). 
En esta situación, tenemos por delante la compleja tarea de reconstruir el pensamiento social otorgando prioridad a los derechos concretos, como lo ha expresado Amartya Sen en diversos trabajos.

Dado que ya no hay lugares (sociales) a los cuales acogerse, cada cual ha de poder abrir y sostener los suyos y generar "lo común", como espacios de resistencia frente al avasallamiento que opera el tecnopoder. Y aquí, en medio de la licuefacción social, la educación social ha encontrado, hasta ahora, dos modalidades de "hacer lugar".

1) El neohigienismo: tal como hemos ya planteado, procede catalogando conjuntos en los que fijará cada elemento. Finalmente, establece un "tratamiento" homogéneo: INTERVENIR en cada conjunto. Hemos apelado a la metáfora "furia entomológica" para designar esta modalidad de adjudicación de lugares residuales y tratamiento de los mismos;

2) La segunda posición, la búsqueda para re-crear dimensiones educacionales en las prácticas profesionales del campo social, nos lleva al último punto de esta exposición para presentar la segunda metáfora.

La idea es que algo, en medio de la fluidez de esta modernidad líquida, precipite. Educar, convocar al sujeto como para que algo de la cultura plural precipite $y$ haga sitio Voy a recurrir a la segunda metáfora ${ }^{14}$ : el delta.

Sabemos algunas cosas: que estamos en un marco de des-atención social, institucional, económica, sometido a la presión clasificatoria. También sabemos que la educación recorta el mundo siempre de manera sesgada, no se puede enseñar todo, según el viejo sueño de Comenius. Sin embargo, en lo que hay que insistir a la manera de Hannah Arendt, es en poner "el mundo" (lo que está entre los seres humanos, los bie-

14. El feliz hallazgo lo debo a un Director de Distrito Educativo de la Provincia de Buenos Aires que sugirió, en una discusión acerca de la categoría modernidad líquida, la idea de delta. La película está disponible en: <http://www.youtube.com/watch?v=1ZOBDVWmvMw>. nes que nos pertenecen): el inter-est. Poner entonces trozos, fragmentos de saberes y los lazos hasta entonces establecidos, a disposición de otros: libros, narraciones diversas. Pues, "paradojalmente, la educación que recorta mundo para poder mostrar algo, lo abre".

Si algo muestra (de las historias, de los pensamientos, del arte), enseña que hay más bienes y que otros encuentros y nuevas invenciones serán posibles. En el paisaje de ruinas que promueven los mercados, la educación pone así en juego, para cada uno, una promesa.

\section{Proponemos pensar:}

Ante la política generalizada de segregación de sujetos y poblaciones, por qué no pensar que sería posible allí, en espacios de deriva a lo peor de cada cual, producir un meandro que abra otro espacio, otro tiempo, otras inclinaciones. Los elementos culturales ninguneados, sustraídos, saqueados pueden ser restituidos; las fronteras infranqueables, atravesadas en nuevos recorridos: la literatura, la música, la tecnología, los saberes, el arte... Materiales aluvionales que se entrecruzan y dan lugar a zonas emergentes, plataformas donde sujetarse para no ser arrastrado al vertedero (BAUMAN, 2005).

Esta posibilidad de abrir mundos es lo propio de la educación, más aún en este momento en que se pretende devaluarla a mera técnica de control en alguna de sus vertientes: operadores de la prevención o la empatía, enseñantes de la inteligencia emocional o subidores de autoestima... Se trata de abrir los guetos conectando a los sujetos con el vasto mundo plural, antes de dar por hecho que ese mundo les está negado. Dejar atrás la desafortunada premisa de Pestalozzi (1775, p.115): "educar al pobre para pobre, pero feliz".

Frente a la des-escolarización / analfabetización de los más jóvenes (¡no vamos a llamarlo (su) fracaso escolar!) Los niños y los adolescentes han de aprender. El neoliberalismo pedagógico sostiene el vaciamiento de la educación en dos categorías: in-educa- 
bles y sobre-educados. Así señala a los que sobran en sus cálculos: los que no son ni serán necesarios y los que ya sirven tal y como están.

Sin embargo, hemos de enfatizar -frente a la mirada extraviada de los adultos de hoy- que los más jóvenes quieren aprender. Los adolescentes hoy nos intimidan... Los padres, al igual que en los inicios de la modernidad sólida, los acusan ante los juzgados. Pero niños, adolescentes y jóvenes pueden aprender y nosotros podemos/debemos enseñarles algo de lo que nos fue dado. Sin duda, ya no son como eran antes, y esto ya lo decían los antiguos griegos ante sus nuevas generaciones.

Los más jóvenes, cada vez, traen algo nuevo; esa novedad interroga a los adultos, los desasosiega, pero también les regala la frescura de un mundo visto con ojos nuevos. No podemos tampoco ignorar que hay una cierta destructividad en juego, necesaria para abrirse camino, para hacerse espacio, no se trata entonces de criminalizar(los). Cada vez, cada nueva generación, ejerce cierta fuerza de destrucción pues se incorpora al viejo mundo en parte para perpetuarlo, en parte para deconstruirlo $y$, en gran parte, para adentrarse en un mundo otro que, cada vez, los adultos no pueden siquiera entrever.

No se trata de focalizar la mirada en lo que nos aparece como ajeno, extranjero, y que nos hace ver al sujeto como inquietante y juzgarlo entonces peligroso, pues allí se abre el cajón entomológico.

\section{EJERCICIOS DE RESISTENCIA Y APERTURA: ESPACIOS CULTURALES EN LAS TRAMAS URBANAS}

Las ciudades son espacios que compartimos. Tema que hoy parece necesario revisar, ante tanta normativa, tanta prohibición, tanta cámara de video vigilando, tanta criminalización de los más jóvenes y de las personas que llegan a las ciudades, procedentes de otras - de aquí y de allá - y a las que se sigue llaman- do inmigrantes, aunque hayan llegado hace ya tiempo. 0 como si migrante, de alguna manera, no lo fuera todo ciudadano.

Hay que plantear el debate acerca de los lugares que estamos dispuestos a abrir en la ciudad, en cada ciudad, para que la ciudadanía sea un ejercicio culturalmente enriquecedor.

Hoy, la labor educativa ha de convocar en plazas, paseos, ramblas y alamedas a actividades culturales que tengan que ver con la lectura, con la música, el teatro, la fotografía, la poesía, el street-art, en fin, con múltiples producciones y bienes culturales capaces de dar lugar a los sujetos en la construcción de espacios de apropiación, creación y pertenencia.

La idea es entrecruzar elementos culturales en redes diversas, para provocar depósitos de distintos materiales, pesados y livianos, que construyan zonas fértiles en las que realizar actividades inicialmente ni siquiera pensables. Y donde los sujetos puedan legitimarse, filiarse, habitar y autorizarse a la cultura plural y al intercambio.

El discurso dominante construye, sin cesar, cajones entomológicos. Pero la fuerza del cauce del agua da paso a geografías nuevas: incontenibles, aluvionales y feraces. Nuestra apuesta es por una Pedagogía social crítica, es decir, “anacrónica”" ${ }^{15}$, que nos permita reconceptualizar mundo y actuar en él "bifurcando"16 el cauce previsto, abriendo brechas.

15. Retomo aquí la idea de Giorgio Agamben (2007): Què vol dir ser contemporani? Barcelona: Arcàdia. "Quien pertenece verdaderamente a su tiempo, quien es verdaderamente contemporáneo, es quien no coincide perfectamente ni se adapta a las pretensiones del mismo y es por ello, en este sentido, inactual; pero también precisamente por eso, y justamente a través de esa desviación y de este anacronismo, que es más capaz que otros de percibir y aferrar su tiempo" [p. 8, traducción propia]. 16. NEGRI, A. Producir lo común. En: AA.VV. (2012): Pensar desde la izquierda. Mapa de un pensamiento crítico para un tiempo en crisis. Madrid: Errata Naturae, p. 147-163. 


\section{REFERENCIAS}

ARENDT, H. La condición humana. Barcelona: Paidos, 1993.

ARENDT, $H$. Entre el pasado y el futuro. Barcelona: Península, 1996.

BAUMAN, Z. La modernidad líquida. Buenos Aires: F.C.E., 2003.

FOUCAULT, M. Vigilar y castigar: el nacimiento de la prisión. Madrid: Siglo XXI, 1981.
PESTALOZZI, J. (1775): Súplica a filántropos y bienhechores para el establecimiento de una alquería donde dar educación y trabajo a niños pobres. EN: QUINTANA CABANAS, J. M. Educación Social. Antología de textos clásicos. Madrid: Narcea, 1994.

TOURAINE, A. Um Novo Paradigma para Compreender o Mundo de Hoje. Lisboa. Instituto Piaget, 2005. 